\title{
CASE REPORT : PRENATAL OCCLUSION OF THE INTERNAL CAROTID ARTERY
}

\author{
BY \\ R. M. CLARK and ERIC A. LINELL \\ From the Department of Pathology and the Division of Neuropathology, University of Toronto
}

The following case is reported as a contribution to our knowledge of the aetiology of " congenital" hemiplegia.

\section{Case Report}

A baby girl, I. (NP. No. 363/53, A. 168/53), died about one and a half hours after her birth on May 13, 1953. The pathological findings were those of erythroblastosis foetalis.

She was the fourth child of an Rh-negative mother and an Rh-positive and homozygous father. The first child was born at full term. The second was stillborn at full term. The mother was given A.C.T.H. before the birth of her third infant, who was born alive at full term but required a replacement transfusion and had a positive Coombs test.

For five weeks before the premature delivery of this fourth infant the mother had received $100 \mathrm{mg}$. of cortisone daily. The pregnancy was normal and there was no antenatal haemorrhage to suggest premature separation of the placenta. Her albumin antibody titre was 1.64 . The infant was born by mid-forceps delivery. The very large, oedematous placenta was manually expressed. The obstetrician considered it otherwise grossly normal.

\section{Necropsy}

Necropsy was performed three hours after death. The infant was immature, weighing only 2,250 g., in spite of severe generalized oedema of subcutaneous and muscular tissues and large effusions in the serous cavities. There was also oedema of the larynx. The placenta was very much enlarged by oedema and weighed $945 \mathrm{~g}$., whereas the weight of the normal placenta is considered to be one-seventh the weight of the infant, which would be about $320 \mathrm{~g}$. in this case. No further gross pathological lesions were found in the placenta to account for its enlargement.

The large spleen weighed $45 \mathrm{~g}$. and contained a small infarct. The liver was also enlarged. The hypertrophied heart weighed $31 \mathrm{~g}$. and its cavities were dilated, possibly due to increased blood volume. The foramen ovale was widely patent and there was a small patent ductus arteriosus. The lungs were atelectatic. The thymus was hypoplastic, weighing only $1 \mathrm{~g}$.

Microscopical examination showed hyperplasia of the bone marrow, with marked proliferation of cells of the erythroblastic series. The liver, kidneys, adrenals, and spleen showed extramedullary haematopoiesis. There were enormous numbers of erythroblasts and a diminution in formation of lymphocytes. The latter finding was considered to account for the hypoplasia of the thymus. The presence of a small infarct in the spleen was confirmed microscopically, but an arterial embolic occlusion could not be demonstrated even by interrupted serial microscopic sections.

A detailed histological examination of the placenta confirmed oedema of the villi. The capillaries contained numerous erythroblasts. A few small areas of infarction, with necrosis of placental villi, were found, a possible site of origin of an embolism of the foetal circulation.

Examination of the Head.- The scalp tissues were oedematous and haemorrhagic. There was subperiosteal and slight extradural haemorrhage in relation to the bones of the cranial vault. There was no subdural haemorrhage and the tentorium cerebelli was grossly normal.

Brain.-This was examined after fixation in $10 \%$ formol-saline for 48 hours. The organ showed prematurity, weighing only $215 \mathrm{~g}$. but the convolutions (Fig. 1) were moderately well formed throughout. There was palpable softening of the cortical distribution of the left middle cerebral artery (Fig. 1), and a horizontal section through the cerebral hemispheres (Fig. 2) confirmed early softening in the complete distribution of this vessel. A dissection of the left internal carotid and middle cerebral arteries showed the lumen of the internal carotid to be occluded by well-defined, white, solid material. This occluding tissue extended to the termination of the internal carotid and for a short distance into its posterior communicating, anterior and middle cerebral branches. The extent of the occlusion was determined by limited serial histological sections and satisfactorily accounted for the softening in the distribution of the left middle cerebral branch. The white colour and the sharp limitation of the occluding tissue were much more suggestive of embolus than of thrombus forming in situ on the vessel wall, as the softening in the middle cerebral distribution was estimated as being of only a few days' duration.

The material occluding the lumina of these vessels does not have the histological characters either of a thrombus formed in situ or of a blood embolus from a distant 
Fig. 1.-Cerebral hemispheres seen from above, showing early cortical softening with a few petechial haemorrhages in the distribution of the left middle cerebral artery.

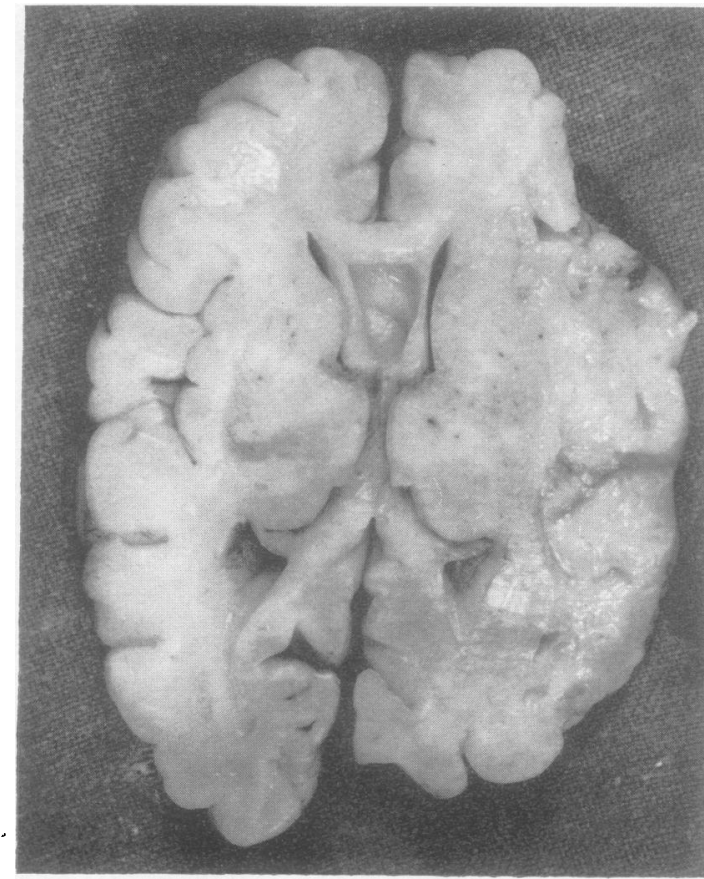

Fig. 2.-Upper cut surface of horizontal section of cerebral hemispheres. This shows softening of a few days' duration in the complete distribution of the left middle cerebral artery (to the right of the photograph). There is a large fifth ventricle.

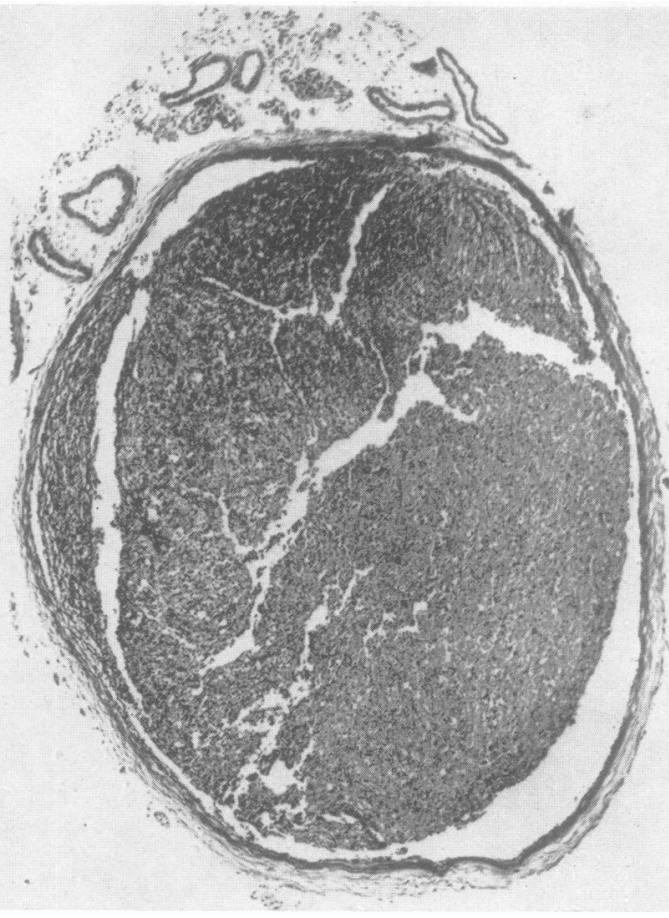

Fig. 3.-Transverse section of the left internal carotid artery at its bifurcation. The lumen of the vessel is occluded by embolic 0 material, the exact origin of which could not be determined.

$8 \frac{\Phi}{3}$

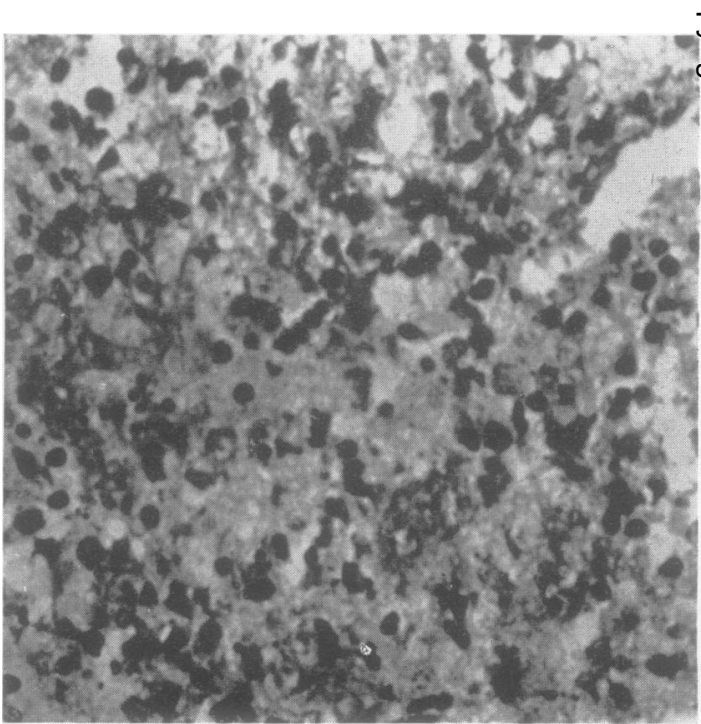

FIG. 4.-Medium-power photomicrograph of the embolic tissue (Fig. 3). It appears to be composed of "dead" cells with degenerating hyperchromatic nuclei. Some of the nuclei are those of erythroblasts from the circulating blood. This occluding material may be dead tissue from the infant's severely diseased placenta. 
focus (Figs. 3 and 4). The main mass of the material appears to be dead tissue, the origin of which cannot be determined. It is infiltrated with moderate numbers of red blood corpuscles, many of which contain nuclei. The vessel wall shows early reactive inflammatory changes, maximum in amount at the point of adhesion of the occluding material to the intima (at bottom of Fig. 3). This suggests the possibility of thrombus formation in situ but such changes could be produced by the pressure of an embolus. The extent of the reaction indicates that the occlusion had been present for at least some days.

The softened cortex and white matter in the left middle cerebral distribution shows the severe vacuolation of early softening. Fully formed lipoid-filled histiocytes are seen in the cortical tissue and white matter, and there are similar microglial cells, filled with lipoid, in the subarachnoid space overlying the softened cortex. Sections stained with Scharlach $\mathbf{R}$ confirm early phagocytosis of fatty degenerative products by microglial cells in the white matter. This would date the softening as being of three days to one week of age.

It seems likely, therefore, that the middle cerebral occlusion occurred, in this case, during the last few days of pregnancy. Embolism appears more probable than thrombosis in situ, and, in the absence of another source, an embolus of infarcted, necrotic placental tissue is suggested.

The infarct found in the spleen was probably also due to arterial embolic occlusion, although an occluded artery could not be found even after detailed microscopical examination.

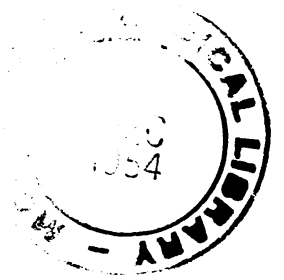

